\title{
Syndromic Surveillance Evaluation of Influenza Activity in At-Risk Sub-Populations
}

\author{
Heather Rubino*, Janet J. Hamilton, Allison B. Culpepper, Hunter Davis, David Atrubin \\ and Melissa Murray Jordan
}

Epidemiology, Florida Department of Health, Tallahassee, FL, USA

\section{Objective}

To determine if emergency department (ED) based syndromic surveillance can be utilized to characterize in near real-time influenza infection in three high-risk populations: a) adults $>65, \mathrm{~b})$ pregnant women, and c) children $<5$.

\section{Introduction}

Near real-time emergency department chief complaint data is accessed through Florida's syndromic surveillance system: Electronic Surveillance System for the Early Notification of Communitybased Epidemics-Florida (ESSENCE-FL). The Florida Department of Health relies heavily upon these data for timely surveillance of influenza and influenza-like illness (ILI). Hospital discharge data available from the Florida Agency for Health Care Administration (AHCA) captures information about influenza-associated ED visits and is considered complete. The delay in receiving the data (up to a year) hinders timely evidence-based decision making during the influenza season. Previous analyses (comparing the complete AHCA hospital discharge data to the ESSENCE-FL ILI syndrome and Influenza sub-syndrome) have shown ESSENCE-FL is a timely, effective tool to monitor influenza activity in the state and that the Influenza sub-syndrome most closely approximates influenza season activity in Florida. Adults $>65$, pregnant women and children $<5$ are at increased risk for morbidity and mortality from influenza infection. This investigation aims to determine if syndromic surveillance can be used to characterize in near real-time influenza infection in adults $>65$, pregnant women, and children $<5$ by comparing ED visits for influenza and ILI in ESSENCE-FL to historical AHCA records of people who incurred ED charges at a Florida hospital with diagnosed influenza.

\section{Methods}

Florida AHCA data for hospital admissions and ED visits from 2008-2014 were queried for patients with diagnosed influenza (ICD9-CM Code: 487 or 488) in the following groups a) adults $>$ $65, \mathrm{~b})$ pregnant women and c) children $<5$. In the AHCA dataset, pregnant women were identified by querying for women aged 14-49 with a diagnosis code of influenza and a pregnancy diagnosis code (ICD9-CM Code: v22 or v23). ESSENCE-FL ED chief complaint data for the three target populations categorized into the ILI syndrome or the Influenza sub-syndrome (or both) for the same time period. In ESSENCE-FL, ED visits in pregnant women were identified using a free text query for "preg" in the chief complaint field among women aged 14-49. A time series by week was used to compare the ESSENCE-FL ILI syndrome data and the Influenza sub-syndrome data to the influenza diagnosis data from AHCA for each of the three target populations. Subsequently, AHCA data were compared to ESSENCE-FL ILI syndrome data and the Influenza sub-syndrome (among each of the three target populations) by week to determine the correlation between the syndromic surveillance categories and AHCA discharge diagnoses.

\section{Results}

In the AHCA data set a) 29,503 adults $>65$, b) 858 pregnant women, and c) 71,807 children $<5$ with influenza diagnoses incurred ED charges from Florida hospitals. In the ESSENCE-FL dataset the ILI syndrome identified: a) 62,015 visits from adults $>65$, b) 1,841 visits from pregnant women, and c) 288,033 visits from children $<5$; use of the Influenza sub-syndrome identified: a) 36,345 visits from adults $>65$, b) 1,217 visits from pregnant women and c) 25,858 visits from children $<5$ years of age. In all three sub-populations, trend (season onset, duration, and end) and volume analyses showed that ED visits categorized into the ESSENCE-FL Influenza subsyndrome best matched the ACHA data for approximating the impact of influenza in the target populations. Correlation analyses found that the ESSENCE-FL Influenza sub-syndrome had higher correlation coefficients than the ILI syndrome when compared to the AHCA data by week (albeit these gains were very small for the adults over 65 and pregnant women groups). None of the queries had a correlation coefficient lower than 0.76 .

\section{Conclusions}

Syndromic surveillance can be used not only to monitor overall influenza trends, but is also effective for timely surveillance and estimation of influenza activity in three target populations: a) adults $>65$, b) pregnant women, and c) children $<5$ in Florida. While rich, complete AHCA data may be up to one year old, which does not allow for timely and informed decision-making and prioritization of resources during influenza season.

\section{Keywords}

influenza; syndromic; at-risk populations

\section{*Heather Rubino}

E-mail: heather.rubino@flhealth.gov 